\title{
Management of Gliomas: Individualized Treatment Options
}

\author{
Presented by Louis Burt Nabors, MD
}

\section{ABSTRACT}

Optimizing treatment for patients with low-grade gliomas should focus on the role of radiation and chemotherapy, as well as the prognostic impact of molecular diagnostics (1p/19q and IDH status). For anaplastic oligodendroglioma, focus should be placed on molecular markers (particularly $1 \mathrm{p} / 19 \mathrm{q}$ status) and combination treatment with chemotherapy (temozolomide or PCV [procarbazine, lomustine, and vincristine]) and radiation. For patients with malignant glioblastomas, the role of methylguanine methyl-transferase (MGMT) methylation status has become increasingly important to treatment decisions. MGMT methylation status should be considered in elderly patients and/or those with low performance status (methylated patients benefit from temozolomide) and a hypofractionated radiation schedule should be used.

J Natl Compr Canc Netw 2020;18(7.5):985-988 doi: $10.6004 /$ jnccn.2020.5008

\section{Impact of Glioma}

Patients with gliomas tend to have a high disease burden compared with patients with other malignancies. At the NCCN 2020 Virtual Annual Conference, according to Louis Burt Nabors, MD, Program Leader for Neuro-Oncology, O'Neal Comprehensive Cancer Center at UAB, and Chair of the NCCN Guidelines Panel for Central Nervous System (CNS) Cancers, "there is a significant need and opportunity for us to do better." Although treatment varies widely according to disease classification, certain factors, particularly molecular markers, methylguanine methyltransferase (MGMT) methylation status, and performance status, should be used to guide treatment decisions.

The median age of individuals diagnosed with a glioblastoma, the most malignant of gliomas, is 56 years in clinical trial populations but 65 years overall, highlighting a potential gap in the research regarding the management of older patients with gliomas, according to Dr. Nabors. "And unfortunately, there's a pretty big difference in survival based on age," he noted. Median overall survival is 17 months from diagnosis for glioblastoma in the general population, compared with 9 months in elderly patients (aged $>65$ years).

According to Dr. Nabors, this particular cancer population is rated high on "years of life lost" due to cancer, an NCI metric used to measure disease burden on patients. Additionally, the public health costs of this disease and its treatment are among the highest in oncology. ${ }^{1}$

The incidence of gliomas spans the age spectrum, from children all the way to older adults. It is the leading cause of cancer death in children (aged 0-14 years), is among the top 3 causes of cancer death for adolescents and young adults (aged 15-39 years), and ranks seventh for cancer deaths among adults aged $\geq 40$ years. $^{2}$

The location of this malignancy in the CNS has a significant impact on patients and their overall performance status, he added. Gliomas have a substantial impact on language functioning in many patients, in terms of both expression and comprehension.

\section{Treating Oligodendrogliomas}

Oligodendrogliomas account for approximately $20 \%$ of adult brain tumors, and most oligodendrogliomas have $1 \mathrm{p} / 19 \mathrm{q}$ chromosome losses. Low-grade oligodendrogliomas tend to be slow-growing tumors, whereas anaplastic oligodendrogliomas (grade III according to WHO classification, IDH-1-mutated, 1p/19q co-deleted) are more aggressive tumors that grow more quickly. "Be attentive to $1 \mathrm{p} / 19 \mathrm{q}$ status, as it's really required now for a diagnosis of anaplastic oligodendroglioma," he advised.

The optimal treatment recommendation for anaplastic oligodendroglioma is a combination of radiotherapy (RT) and chemotherapy, based on results of 2 clinical trials: RTOG 9402, ${ }^{3}$ which used an adjuvant chemotherapy approach, and EORTC $26951,{ }^{4}$ which used neoadjuvant chemotherapy.

Both trials evaluated PCV (procarbazine, lomustine, vincristine) plus $R T$ versus $R T$ alone in patients with $1 p / 19 q$ co-deleted anaplastic gliomas. According to Dr. Nabors, most patients in both studies had trouble completing the number of cycles prescribed due to PCV-associated toxicities. 
"The results of these studies were strikingly similar in that both showed a survival advantage for the groups that received chemotherapy and RT," he said, "somewhat confirming a role for chemotherapy and RT early in the disease course."

The NCCN Guidelines for CNS recommend consideration of a clinical trial for all patients with glioma. Focusing on patients with $1 \mathrm{p} / 19 \mathrm{q}$ co-deleted anaplastic oligodendrogliomas, standard RT and neoadjuvant or adjuvant PCV are considered the next option, followed by consideration of the role of temozolomide, he said.

\section{Implications for Patient Care}

According to Dr. Nabors, RT alone is no longer considered adequate for patients with anaplastic oligodendrogliomas with $1 \mathrm{p} / 19 \mathrm{q}$ co-deletion. Existing data support first-line treatment with RT and chemotherapy, but the optimal treatment paradigm has not yet been established (chemotherapy $\rightarrow$ RT or RT $\rightarrow$ chemotherapy).

The enduring debate continues regarding the optimal choice of chemotherapy (temozolomide vs PCV). Currently, both remain treatment options in the NCCN Guidelines for CNS, but the ongoing CODEL study will attempt to settle the question by randomizing patients to receive RT and 1 of these 2 chemotherapy regimens (ClinicalTrials.gov identifier: NCT04407039). "So keep the CODEL study in mind for this patient population," he advised.

\section{Glioma Grading and Natural History}

The category of low-grade glioma includes astrocytomas and oligodendrogliomas. Dr. Nabors highlighted some distinguishing features of low-grade gliomas, emphasizing that they are not benign; they will progress over time, and can be highly heterogeneous in their behavior (Figure 1).

"It's increasingly important that we recognize the need to more uniformly obtain molecular diagnostics, including IDH-1 status and $1 \mathrm{p} / 19 \mathrm{q}$ status," said Dr. Nabors. He noted that the molecular classification of low-grade glioma associated with the worst outcomes is IDH-1/2 wild-type, because these tend to represent a more aggressive glioma that will progress rapidly to glioblastoma.

\section{Treatment of Low-Grade Gliomas}

According to Dr. Nabors, surgical resection plays an important role in patients with low-grade gliomas. Standard treatment options for diffuse astrocytomas (WHO grade II) include surgery alone, or surgery followed by RT or chemotherapy, or a combination.

High-risk patients with grade II glioma who had less than gross total tumor resection or who are aged $>40$ years should receive RT followed by PCV, based on results from the RTOG 9802 study in which the combination prolonged both progression-free and overall survival compared with RT alone. ${ }^{5}$ Rates of 5- and 10-year survival were increased by $9 \%$ and $20 \%$, respectively, making this the first prospective study ever to demonstrate a treatment-related increase in survival in patients with grade II glioma. ${ }^{6}$

Patients in the trial who were aged $<40$ years and had gross total resection were deemed low risk and were observed. "But I don't know that I'd adhere to that age cutoff of 40 years religiously," he noted, adding that performance status should also be considered.

Dr. Nabors emphasized the prognostic impact of the molecular diagnostics of these malignancies, particularly $1 \mathrm{p} / 19 \mathrm{q}$ and IDH status. Low-grade gliomas fall into 1 of 3 molecular diagnostic categories: 1p/19q co-deletion and IDH mutation; IDH mutation and no $1 \mathrm{p} / 19 \mathrm{q}$ co-deletion; or neither IDH mutation nor 1p/19q co-deletion. ${ }^{7}$

"Be alert to the IDH wild-type group," he reiterated. "The molecular alterations in this group are more consistent with a primary glioblastoma. Although [they constitute] a small fraction, the clinical behavior of this group is very aggressive, and should be treated and considered more as a glioblastoma than a low-grade glioma."

\section{Treatment of Malignant Astrocytomas}

The category of malignant astrocytomas includes anaplastic astrocytoma, a grade III malignant glioma that occurs in a slightly younger population (and is slightly less aggressive with somewhat better prognosis than glioblastoma multiforme), as well as glioblastoma multiforme (WHO grade IV).

Glioblastoma is the most aggressive type of glioma, "harboring all of the histopathologic features associated with bad behavior," he said. It is most common in older adults (peak age, 55-65 years), and is characterized by rapid growth (size may double every 10 days).

According to Dr. Nabors, there are 2 ways to "arrive" at a glioblastoma. The vast majority are primary, and harbor certain sets of molecular alterations, but there is also a stepwise route, occurring in a minority of patients.

"We're probably moving more toward using the term, 'anaplastic astrocytoma grade IV,' rather than 'secondary glioblastoma,' which is quite traumatizing for patients and families," he said. "Even though glioblastoma is often the ultimate event in this stepwise sequence, the outcomes are different, the patient population is different, and as a result, some of the treatment options may emerge in the near future to be different, so just be alert to that."

Based on a pivotal 2005 study, ${ }^{8}$ treatment recommendations for glioblastoma include RT and concurrent 


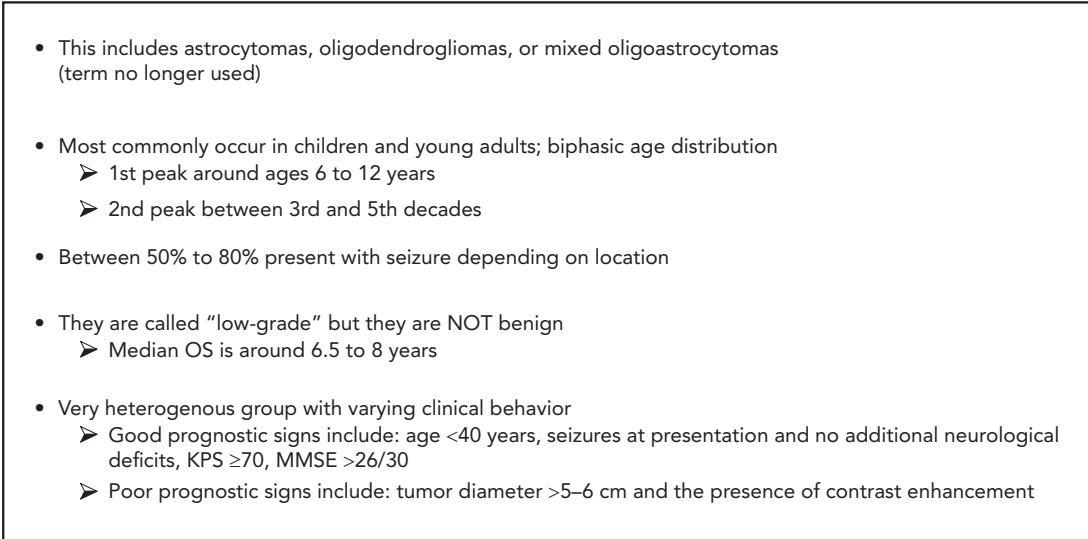

Figure 1. WHO grade II low-grade gliomas.

Abbreviations: KPS, Karnofsky performance status; MMSE, mini-mental state examination; OS, overall survival.

chemotherapy with temozolomide, or a clinical trial where available.

\section{Treating Older Patients With Glioblastoma}

Clinical trials for newly diagnosed glioblastoma clearly demonstrate the increasing role for determining MGMT methylation status and highlight the importance of tailoring treatment to this information. A companion manuscript to the trial conducted by Stupp et $\mathrm{al}^{8}$ examined the role of MGMT gene silencing as a predictor of benefit from chemotherapy with temozolomide, and found that patients with glioblastoma containing a methylated MGMT promoter benefited from temozolomide, whereas those who had an unmethylated MGMT promoter did not demonstrate nearly as great a benefit. " "But this has led to some struggles in managing our older patients with glioblastoma," he noted.
In addition to factors such as more aggressive biology, limitations to performance and functional status, limited survival expectations, frailty (including the aging brain being less tolerant to surgery, RT, and chemotherapy) and comorbidities, population-based practice patterns show less use of chemotherapy in the older population, even though up to $40 \%$ have MGMT promoter methylation, equal to their younger counterparts.

Although the survival benefit of RT and temozolomide was previously established in patients aged $<65$ years, the benefit of adding temozolomide to a shorter course of RT was unknown in older patients until the publication of a 2017 study. ${ }^{10}$ In this trial of elderly patients (aged $>65$ years) with newly diagnosed glioblastoma, the addition of temozolomide to short-course RT resulted in longer survival than short-course RT

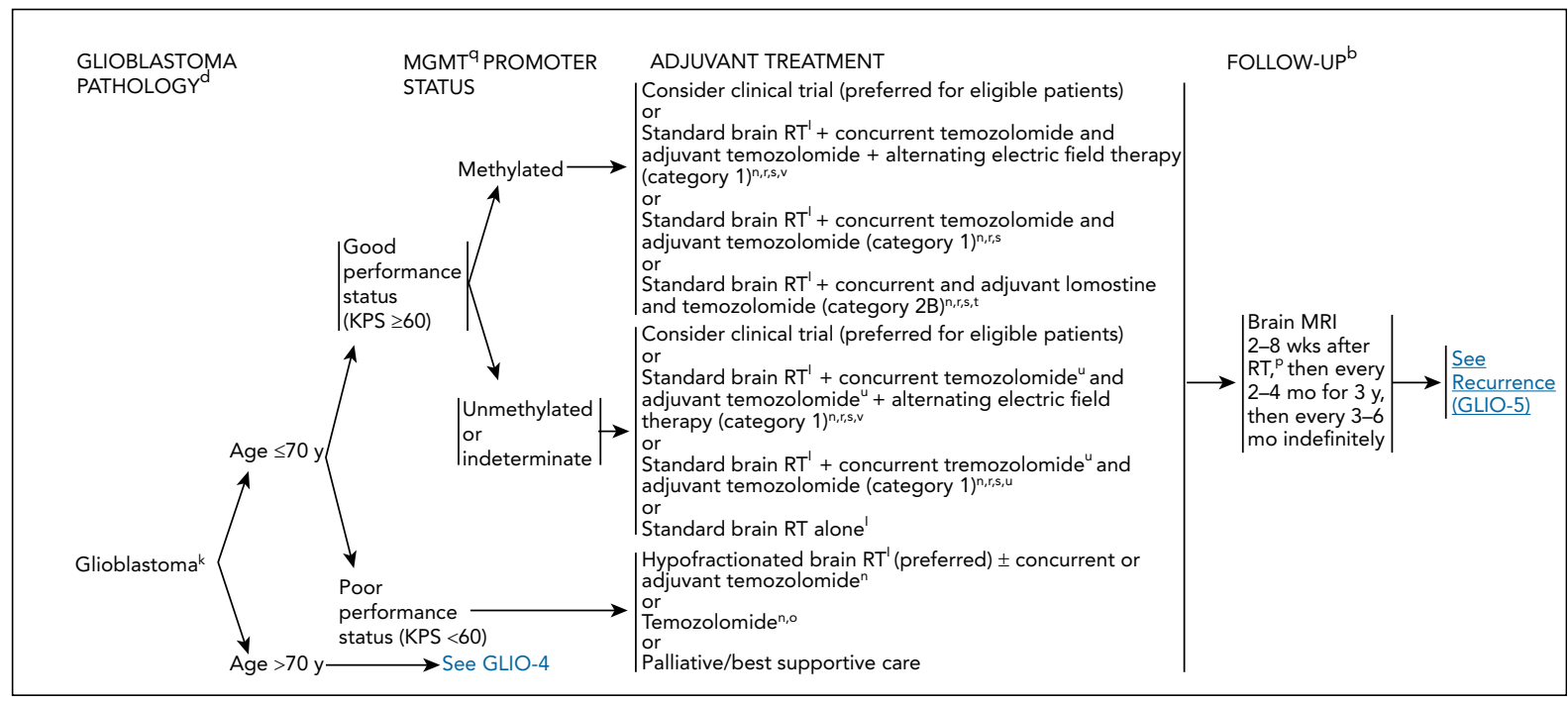

Figrue 2. NCCN Clinical Practice Guidelines in Oncology for Central Nervous System Cancers: Anaplastic Gliomas/Glioblastoma. Version 2.2020. CNCCN. All rights reserved. 
alone. And when patients were stratified according to MGMT status, there was a clear statistically significant benefit of RT plus temozolomide in methylated patients, with much less benefit seen in unmethylated patients.

"I do believe it's critically important to factor MGMT status into your treatment decisions. For methylated patients, I think there's a clear advantage to the use of temozolomide," he said. "For unmethylated patients, we provide an opportunity in the guidelines to avoid giving temozolomide: (1) it's toxic; (2) it's expensive; and (3) it is a very lymphotoxic drug."

Treatment for elderly patients or those with low performance status who have glioblastoma should be guided by MGMT methylation status, and should follow a hypofractionated radiation schedule, he added (Figure 2).

Dr. Nabors concluded by pointing out that within the 1 to 6 months following RT, enhancement and even edema will often look worse on an MRI. However, the criteria for progression during that time frame would require clear evidence of disease outside of the radiation field, he said, adding that providers should familiarize themselves with the Response Assessment in NeuroOncology (RANO) criteria. $^{11}$

"Radiologic interpretation is calling it as they see it. If they're not aware of the timing related to therapy, an increase in enhancement and in edema would certainly be interpreted as consistent with tumor progression," he cautioned. "But in fact, it's probably more likely related to the treatment of the disease, rather than the disease itself."

Disclosures: Dr. Nabors has disclosed that he is a scientific advisor for AbbVie, Inc., BTG Pharmaceuticals, Karyopharm Therapeutics, Blue Earth Diagnostics, and Kiyatec.

Correspondence: Louis Burt Nabors, MD, O'Neal Comprehensive Cancer Center at UAB, 510 20th Street South, FOT 1020, Birmingham, AL 35294. Email: bnabors@uab.edu

\section{References}

1. Yabroff KR, Lund J, Kepka D, et al. Economic burden of cancer in the United States: estimates, projections, and future research. Cancer Epidemiol Biomarkers Prev 2011;20:2006-2014.

2. Ostrom QT, Gittleman H, Xu J, et al. CBTRUS statistical report: primary brain and other central nervous system tumors diagnosed in the United States in 2009-2013. Neuro Oncol 2016;18(Suppl 5):v1-75.

3. Cairncross G, Wang M, Shaw E, et al. Phase III trial of chemoradiotherapy for anaplastic oligodendroglioma: long-term results of RTOG 9402. J Clin Oncol 2013;31:337-343.

4. van den Bent MJ, Brandes AA, Taphoorn MJB, et al. Adjuvant procarbazine, lomustine, and vincristine chemotherapy in newly diagnosed anaplastic oligodendroglioma: long-term follow-up of EORTC Brain Tumor Group Study 26951. J Clin Oncol 2013;31:344-350.

5. Shaw EG, Wang M, Coons SW, et al. Randomized trial of radiation therapy plus procarbazine, lomustine, and vincristine chemotherapy for supratentorial adult low-grade glioma: initial results of RTOG 9802. J Clin Oncol 2012:30:3065-3070.
6. Buckner JC, Shaw EG, Pugh SL, et al. Radiation plus procarbazine, CCNU, and vincristine in low-grade glioma. N Engl J Med 2016;374 1344-1355.

7. Cancer Genome Atlas Research Network, Brat DJ, Verhaak RGW, et al. Comprehensive, integrative genomic analysis of diffuse lower-grade gliomas. N Engl J Med 2015;372:2481-2498.

8. Stupp R, Mason WP, van den Bent MJ, et al. Radiotherapy plus concomitant and adjuvant temozolomide for glioblastoma. N Engl J Med 2005;352:987-996.

9. Hegi ME, Diserens A, Gorlia T, et al. MGMT gene silencing and benefit from temozolomide in glioblastoma. N Engl J Med 2005;352:997-1003.

10. Perry JR, Laperriere N, O'Callaghan CJ, et al. Short-course radiation plus temozolomide in elderly patients with glioblastoma. N Engl J Med 2017; 376:1027-1037.

11. Wen PY, Macdonald DR, Reardon DA, et al. Updated response assessment criteria for high-grade gliomas: Response Assessment in NeuroOncology Working Group. J Clin Oncol 2010;28:1963-1972. 